\title{
Tandem mass spectrometry, but not T-cell receptor excision circle analysis, identifies newborns with late-onset adenosine deaminase deficiency
}

\author{
Giancarlo la Marca, PharmSc, ${ }^{\text {a,b }}$ Clementina Canessa, MD, ${ }^{\text {b,c }}$ Elisa Giocaliere, BSc, ${ }^{\text {a,b }}$ Francesca Romano, BSc, \\ Marzia Duse, MD, ${ }^{d}$ Sabrina Malvagia, BSc, ${ }^{\text {a,b }}$ Francesca Lippi, MD, ${ }^{\text {b,c }}$ Silvia Funghini, BSc, ${ }^{\text {a,b }}$ Leila Bianchi, MD, $^{\text {b,c }}$ \\ Maria Luisa Della Bona, PharmSc, ${ }^{\text {a,b }}$ Claudia Valleriani, BSc, ${ }^{b, c}$ Daniela Ombrone, BSc, ${ }^{\text {a,b }}$ Maria Moriondo, BSc, PhD, ${ }^{\text {b,c }}$ \\ Fabio Villanelli, BSc, ${ }^{a, b}$ Carsten Speckmann, MD, ${ }^{e}$ Stuart Adams, PhD, ${ }^{f}$ Bobby H. Gaspar, MD, PhD, ${ }^{f}$ \\ Michael Hershfield, MD, ${ }^{\mathrm{g}}$ Ines Santisteban, PhD, ${ }^{\mathrm{g}}$ Lynette Fairbanks, BSc, PhD, ${ }^{f}$ Giovanni Ragusa, MD, ${ }^{d}$ \\ Massimo Resti, MD, ${ }^{\mathbf{b}, \mathbf{c}}$ Maurizio de Martino, MD, ${ }^{\mathbf{b}, \mathbf{c}}$ Renzo Guerrini, MD, ${ }^{\mathbf{h}}$ and Chiara Azzari, MD, PhD ${ }^{\mathbf{b}, \mathbf{c}}$ Florence and \\ Rome, Italy, Freiburg, Germany, London, United Kingdom, and Durham, NC
}

Background: Adenosine deaminase (ADA)-severe combined immunodeficiency (SCID) is caused by genetic variants that disrupt the function of ADA. In its early-onset form, it is rapidly fatal to infants. Delayed or late-onset ADA-SCID is characterized by insidious progressive immunodeficiency that leads to permanent organ damage or death. Quantification of T-cell receptor excision circles (TRECs) or tandem mass spectrometry (tandem-MS) analysis of dried blood spots (DBSs) collected at birth can identify newborns with early-onset ADASCID and are used in screening programs. However, it is not clear whether these analyses can identify newborns who will have delayed or late-onset ADA-SCID before symptoms appear. Objective: We performed a retrospective study to evaluate whether tandem-MS and quantitative TREC analyses of DBSs could identify newborns who had delayed-onset ADA-SCID later in life. Methods: We tested stored DBSs collected at birth from 3 patients with delayed-onset ADA-SCID using tandem-MS (PCT EP2010/070517) to evaluate levels of adenosine and $2^{\prime}$ deoxyadenosine and real-time PCR to quantify TREC levels. We also analyzed DBSs from 3 newborns with early-onset ADASCID and 2 healthy newborn carriers of ADA deficiency. Results: The DBSs taken at birth from the 3 patients with delayed-onset ADA-SCID had adenosine levels of 10, 25,

\footnotetext{
From 'the Department of Pharmacology, ${ }^{\mathrm{b}}$ Anna Meyer Children's University Hospital, and ${ }^{h}$ the Clinic of Pediatric Neurology, Department of Neurology, University of Florence; 'the Department of Women and Children's Health, University of Florence; 'the Department of Pediatrics and Pediatric Neuropsychiatry, University of Rome "La Sapienza"; 'Zentrum für Kinderheilkunde und Jugendmedizin, Centrum fuer Chronische Immundefizienz-Universitaet Freiburg; ${ }^{\mathrm{f}}$ the Molecular Immunology Unit, UCL Institute of Child Health, London; and ${ }^{g}$ the Department of Medicine, Duke University Medical Center, Durham.

Supported in part by a donation from Famiglia Cassigoli (C.A.) and a grant from the University of Florence (C.A. and G.1.M.), the Anna Meyer Children's University Hospital (M.R.), and the Tuscany (Italy) region (C.A., G.1.M., and M.R.). Sigma-Tau Pharmaceuticals provided grant support to M.H. and I.S.

Disclosure of potential conflict of interest: B. H. Gaspar has consultant arrangements with Enzon. I. Santisteban and M. Hershfield have received grants from Sigma-Tau Pharmaceuticals. The rest of the authors declare that they have no relevant conflicts of interest.

Received for publication January 10, 2012; revised August 23, 2012; accepted for publication August 24, 2012.

Corresponding author: Chiara Azzari, MD, PhD, Department of Pediatrics, University of Florence, Anna Meyer Children's University Hospital, Viale Pieraccini 24, 50139 Firenze, Italy. E-mail: chiara.azzari@unifi.it.

0091-6749/\$36.00

(C) 2013 American Academy of Allergy, Asthma \& Immunology

http://dx.doi.org/10.1016/j.jaci.2012.08.054
}

and $19 \mu \mathrm{mol} / \mathrm{L}$ (normal value, $<1.5 \mu \mathrm{mol} / \mathrm{L}$ ) and $2^{\prime}$ deoxyadenosine levels of $0.7,2.7$, and $2.4 \mu \mathrm{mol} / \mathrm{L}$ (normal value, $<0.07 \mu \mathrm{mol} / \mathrm{L}$ ); the mean levels of adenosine and $2^{\prime}$ deoxyadenosine were respectively 12.0- and 27.6-fold higher than normal values. DBSs taken at birth from all 3 patients with delayed-onset ADA deficiency had normal TREC levels, but TRECs were undetectable in blood samples taken from the same patients at the time of diagnosis.

Conclusion: Tandem-MS but not TREC quantification identifies newborns with delayed- or late-onset ADA deficiency. (J Allergy Clin Immunol 2012;

Key words: Adenosine deaminase, severe combined immunodeficiency, newborn screening, tandem-mass-spectrometry, late-onset, delayed-onset, Adenosine deaminase-severe combined immunodeficiency, T-cell receptor excision circle, genetics, inherited disorder

Severe combined immunodeficiency (SCID) is a group of rare diseases characterized by recurrent serious or often fatal infections. ${ }^{1}$ Characteristics of SCID meet the following criteria necessary for this disease to be included in a neonatal screening program ${ }^{2}$ : high mortality and morbidity, an ability to be cured if it is recognized early, an early presymptomatic phase, availability of simple and inexpensive tests for disease markers, and defined and available treatments. ${ }^{3}$

SCID can be identified by using PCR analysis of dried blood spots (DBSs) for T-cell receptor excision circles (TRECs). ${ }^{4}$ This assay has a rate of indeterminate or false-positive results similar to that of other newborn screening tests, ${ }^{4,5}$ although the rate of indeterminate results is higher among particular subgroups, such as premature infants. ${ }^{4,5}$ The costs might be too high for routine use in many countries.

Adenosine deaminase (ADA) deficiency is an autosomal recessive disorder in which genetic variants disrupt the activity or production of ADA. This deficiency disrupts the purine salvage pathway and also results in an accumulation of deoxyadenosine in cells, which blocks the development of T and B cells of the immune system. ADA deficiency accounts for approximately $15 \%$ to $20 \%$ of cases of SCID, although this might be an underestimate because many infants with this disorder die before a diagnosis is made. ${ }^{6,7}$

Both delayed- and late-onset ADA-SCID are characterized by progressive immunodeficiency that leads to recurring severe infections, immune dysregulation, and organ damage associated with metabolite accumulation. ${ }^{8,9}$ The incidence of late-onset ADA deficiency is also likely to be underestimated, even more than that of early-onset ADA-SCID. ${ }^{8}$ However, excellent 
Abbreviations used

ADA: Adenosine deaminase

dAXP: 2'-Deoxydenosine nucleotides

DBS: Dried blood spot

SCID: Severe combined immunodeficiency

Tandem-MS: Tandem mass spectrometry

TREC: T-cell receptor excision circle

therapeutic options are available for all forms of ADA deficiency if diagnosed at an early stage. ${ }^{10,11}$

We have recently shown that it is possible to identify patients with ADA-SCID using tandem mass spectrometry (tandem-MS) on DBSs collected at birth during routine newborn screening procedures at a low $\operatorname{cost}(0.01 €$ per test $) .{ }^{12}$ An expanded newborn screening including more than 40 metabolic diseases is now performed by using tandem-MS in many countries in the world, so that millions of children worldwide are screened each year for those diseases. ${ }^{13}$ In the Tuscany region of Italy, ADA-SCID has been added to the panel of diseases for which tandem-MS screens. A pilot population-based screening program began in 2010 that uses TREC and tandem-MS analyses to identify newborns with SCID.

We investigated whether analysis of DBSs collected during newborn screening procedures can identify patients with delayedor late-onset ADA deficiency by using TREC analysis, tandemMS, or both.

\section{METHODS \\ Patients}

Patients 1-3. We retrieved stored DBSs that were collected at birth from 3 patients (P1-P3) who were given diagnoses of delayed-onset ADASCID when they were 7, 4, and 3 years old, respectively. The patients presented with upper and lower airway diseases, ranging from recurrent otitis and pneumonia to toxic alveolitis, since they were 2 years old. DBSs were analyzed by using tandem-MS and TREC analysis. Patient P1 is described in the article by Speckmann et al. ${ }^{14}$

Patient 4. The expanded newborn screening performed in Tuscany using tandem-MS identified a DBS in which adenosine and 2'deoxyadenosine were increased. The levels of the 2 metabolites were approximately 50 -fold lower than those found in patients with early-onset ADA-SCID ${ }^{12}$ but were still 4- and 17-fold greater, respectively, than those of subjects without this disorder. TREC levels were normal.

To exclude a false-positive result, after confirmation of the results using the same DBS, the recall procedure was started, and patient P4 was invited to the Pediatric Immunology Department (Meyer Hospital, Florence, Italy) for clinical and biochemical assessment. She was the first child of her parents, who were not related. The mother had an uneventful pregnancy and delivery, and patient P4's birth weight was $3150 \mathrm{~g}$. On her first and second visits to the Pediatric Immunology Department (5 and 25 days old), patient P4 was in excellent condition.

P4 was tested for adenosine and 2'-deoxyadenosine with tandem-MS analysis on DBS, whole-blood and urine samples collected at different times during the first 7 months of life. We analyzed ADA activity, immunoglobulin levels, lymphocyte subsets and proliferation, and the sequence of the $A D A$ gene in the patient and her parents. Patient P4 was also assessed for levels of 2 -deoxydenosine nucleotides (dAXP). Activity of the mutated enzyme expressed in Escherichia coli was evaluated. ${ }^{8}$

\section{Control subjects}

On approval of the institutional review board, we performed tandem-MS and TREC analyses of DBSs collected at birth from 2 newborns who carried

$A D A$ variants associated with SCID (siblings of 2 patients with early-onset ADA-SCID) and 3 patients with early-onset ADA-SCID. Whole-blood samples from patients with ADA-SCID and their healthy carrier siblings had been analyzed for ADA activity.

\section{Tandem-MS analysis of DBSs and urine samples}

Samples were prepared for routine screening tests, as previously described ${ }^{15}$ with the exception of the butylation procedure, which was not carried out. The method for identifying ADA metabolites in DBSs has been patented (PCT EP2010/070517) and validated by using 12,000 DBSs from healthy newborns. ${ }^{12}$ Normal values of adenosine are less than $1.5 \mu \mathrm{mol} / \mathrm{L}$, and 2 -deoxyadenosine is not detectable in DBSs collected at birth from newborns without ADA deficiency. The method produced no false-positive results in 50,000 screened newborns, with no difference between full-term or premature births. Purines were quantified in DBSs after subtracting background noise, as determined from a blank spot. Adenosine and 2'-deoxyadenosine levels were measured in urine samples by using a validated high-pressure liquid chromatography tandem-MS method. ${ }^{16}$

\section{Assays for lymphocyte subsets and proliferation, ADA activity, and dAXP levels}

Lymphocyte subsets and proliferation were analyzed by using standard cytofluorimetric methods. Immunoglobulin levels were evaluated by using standard immunometric assays. ADA activity was evaluated with 3 different methods. Method A was performed on hemolysates of whole-blood samples, combining the activity detected in red and white cells. Reference values for method A were as follows: less than $0.3 \mathrm{U} / \mathrm{grams}$ of hemoglobin $(\mathrm{gHb})$ for early-onset ADA-SCID, 0.4 to $0.8 \mathrm{U} / \mathrm{gHb}$ for delayed- or late-onset ADA deficiency (or carriers of $A D A$ variants associated with disease), and 0.8 to 2.5 $\mathrm{U} / \mathrm{gHb}$ for control subjects (normal values). Method B measured ADA activity in extracts of separated erythrocytes or separated white blood cells; this highpressure liquid chromatography-based assay was performed as previously described.$^{17}$ Method $\mathrm{C}$ was a radiochemical thin-layer chromatographic assay. ${ }^{18}$ Normal values for ADA activity in erythrocytes and white blood cells are $63.0 \pm 41.4$ and $1197 \pm 516 \mathrm{nmol} / \mathrm{h} / \mathrm{mg}$ protein, respectively. Untreated patients with new diagnoses of early-onset ADA-SCID and those with delayedand late-onset ADA-SCID usually have less than $1 \%$ of the normal activity of ADA in red and white blood cells. Patients with partial phenotypes lack or have very low ADA activity in red blood cells but have $5 \%$ to $70 \%$ of the normal level of ADA activity in nucleated cells. ${ }^{8,19,20}$

Total adenosine and 2'-deoxyadenosine nucleotide (AXP and dAXP, respectively) levels in erythrocytes were measured in patient $\mathrm{P} 4$, as previously described. ${ }^{8}$ The normal concentration of dAXP is $0.002 \mathrm{nmol} / \mathrm{mL}$ packed red blood cells (erythrocytes). dAXP was also expressed as the percentage of total adenosine nucleotides to compare levels of dAXP that were measured at different times or from different patients: $\% \mathrm{dAXP}=[\mathrm{dAXP}] \times 100 /$ [AXP + dAXP]; the normal value is less than $0.2 \%$. dAXP levels in untreated patients with ADA-SCID vary with phenotype, as previously reported. ${ }^{8}$

\section{TREC analysis}

DNA was extracted from a single 3.2-mm punch of the DBSs by using the QIAamp DNA Mini kit (Qiagen, Hilden, Germany) and analyzed by using real-time quantitative PCR for TRECs and $\beta$-actin, as previously described. ${ }^{21}$ TREC levels were normalized per microliter of blood, assuming that the sample contains approximately $3 \mu \mathrm{L}$ of whole blood. Calibration curves were generated by means of 10 -fold serial dilution of plasmids that contain TREC and $\beta$-actin sequences. Diagnostic cutoff scores were established at $25 \mathrm{TRECs} / \mu \mathrm{L}$.

DBSs had been collected at birth during routine newborn screening procedures and stored. DBSs were collected in the region of birth: Germany for patient $\mathrm{P} 1{ }^{14}$ and Italy for patients $\mathrm{P} 2$ to $\mathrm{P} 4$. When possible, DBSs were also collected at diagnosis (Tables I and II). Stored DBSs were retrieved, and TREC and tandem-MS analyses were performed for each patient by using the same DBS. 


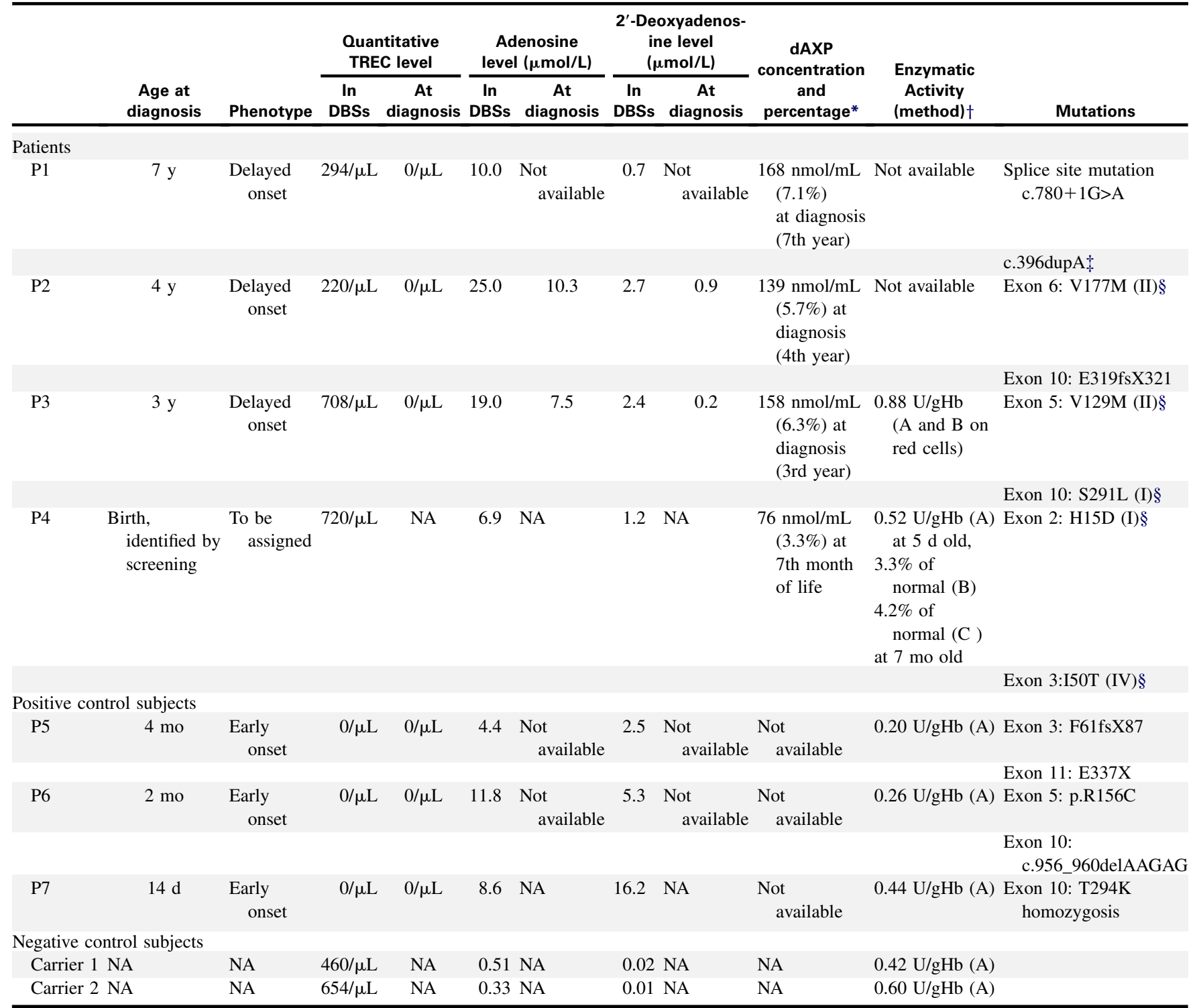

Notes: All DBSs were collected at birth during a routine newborn screening procedure. TREC and tandem-MS analysis were performed on the same DBSs. NA, Not applicable. $\%$ dAXP $=[\mathrm{dAXP}] \times 100 /[\mathrm{AXP}+\mathrm{dAXP}]$.

$†$ For method A, reference values are the following less than $0.3 \mathrm{U} / \mathrm{gHb}$ for patients with early-onset ADA-SCID, 0.4 to $0.8 \mathrm{U} / \mathrm{gHb}$ for patients with delayed- or late-onset disease or carriers, and 0.8 to $2.5 \mathrm{U} / \mathrm{gHb}$ for subjects without disease (control subjects, normal values). For methods B and C, patients with early-, delayed-, or late-onset disease have usually less than $1 \%$ of normal ADA activity in red and white blood cells; ADA enzymatic activity values of $5 \%$ to $70 \%$ of normal values are present in the partial phenotype. $\$$ Speckmann et al. ${ }^{14}$

$\S$ ADA activity group, according to Arredondo-Vega et al. ${ }^{8}$

\section{RESULTS}

\section{Tandem-MS analysis}

Adenosine levels in DBSs from patients with delayed-onset ADA-SCID (P1-P3) were 10, 25, and $19 \mu \mathrm{mol} / \mathrm{L}$, respectively (normal value, $<1.5 \mu \mathrm{mol} / \mathrm{L}$ ), and levels of $2^{\prime}$-deoxyadenosine were $0.7,2.7$, and $2.4 \mu \mathrm{mol} / \mathrm{L}$ (normal value, $<0.07 \mu \mathrm{mol} / \mathrm{L}$; Table I), respectively; the levels were in the same range of those measured for patient P4 (Fig 1,C). The mean adenosine and 2'deoxyadenosine levels in patients P1 to P3 were 12.0- and 27.6fold higher, respectively, than the mean normal values obtained in 50,000 non-ADA-deficient newborns. 2'-Deoxyadenosine levels in patients P1 to P3 and patient P4 were lower than those previously reported by our group for 3 different patients with early-onset ADA-SCID (P5-P7; 2.5, 5.3, and $16.2 \mu \mathrm{mol} / \mathrm{L}$ ), ${ }^{12}$ although the difference in levels was not statistically significant. On the other hand, adenosine levels were greater in patients P1 to $\mathrm{P} 3$ compared with those seen in patients with early-onset disease (P5-P7), without reaching statistical significance (Table I). No difference in the levels of adenosine and $2^{\prime}$-deoxyadenosine was present in the same patient over time (Fig 2).

Tandem-MS analysis of the DBSs collected at birth from patient $\mathrm{P} 4$ revealed high adenosine $(6.9 \mu \mathrm{mol} / \mathrm{L})$ and $2^{\prime}$-deoxyadenosine $(1.2 \mu \mathrm{mol} / \mathrm{L}$; Fig $1, C)$ levels; these were $4.6-$ and 17.1-fold higher, respectively, than the upper limit found in DBSs from healthy newborns (Fig 1,A) but significantly lower than those for patients with early-onset ADA-SCID (Fig 1, B). 
TABLE II. Results from tests of follow-up analysis of patient P4

\begin{tabular}{|c|c|c|c|c|c|c|c|}
\hline Test date & $\begin{array}{l}\text { TREC levels } \\
\text { in DBSs }\end{array}$ & $\begin{array}{c}\text { Adenosine } \\
\text { levels in } \\
\text { DBS } \\
\text { ( } \mu \mathrm{mol} / \mathrm{L} \text { ) }\end{array}$ & $\begin{array}{c}2 \text { '-Deoxyadenosine } \\
\text { levels in DBSs } \\
(\mu \mathrm{mol} / \mathrm{L})\end{array}$ & $\begin{array}{c}\text { dAXP } \\
(\mathrm{dAXP} / \mathrm{AXP} \%)^{*}\end{array}$ & $\begin{array}{l}\text { Enzymatic } \\
\text { activity in } \\
\text { red cells }\end{array}$ & $\begin{array}{c}\text { Enzymatic activity } \\
\text { in white blood cells } \\
\text { (method) }\end{array}$ & $A D A$ variants \\
\hline At birth & $720 / \mu \mathrm{L}$ & 6.9 & 1.23 & - & - & - & $\begin{array}{l}\text { Exon 2: encodes H15D } \\
\text { Exon 3: encodes I50T }\end{array}$ \\
\hline At 5th day & $783 / \mu \mathrm{L}$ & 3.8 & 0.22 & - & Absent & $0.52 \mathrm{U} / \mathrm{gHb}(\mathrm{A}) \dagger$ & \\
\hline At 25 th day & $628 / \mu \mathrm{L}$ & 0.22 & 0.04 & - & - & - & \\
\hline At 3rd month & $694 / \mu \mathrm{L}$ & 1.58 & 0.08 & - & - & - & \\
\hline At 7 th month & $746 / \mu \mathrm{L}$ & 1.54 & 0.09 & $\begin{array}{c}76 \mathrm{nmol} / \mathrm{mL} \\
(3.3 \%)\end{array}$ & Absent & $\begin{array}{l}3.3 \% \text { of normal (B) } \\
4.2 \% \text { of normal (C) }\end{array}$ & \\
\hline
\end{tabular}

$* \% \mathrm{dAXP}=[\mathrm{dAXP}] \times 100 /[\mathrm{AXP}+\mathrm{dAXP}]$.

$\dagger$ Method A was performed on hemolysates of whole blood, combining activities of red and white cells.

Results of analysis of the DBS from patient P4 were confirmed in an analysis performed on the fifth day of life, on a second DBS (adenosine level, $3.8 \mu \mathrm{mol} / \mathrm{L} ; 2$ '-deoxyadenosine level, 0.22 $\mu \mathrm{mol} / \mathrm{L}$; Fig $1, C)$, and on a urine sample $(2.19 \mathrm{mmol} / \mathrm{mol}$ creatinine of adenosine [normal value, $<0.33 \mathrm{mmol} / \mathrm{mol}$ creatinine] and $3.1 \mathrm{mmol} / \mathrm{mol}$ creatinine of $2^{\prime}$-deoxyadenosine [normal value, $<0.01 \mathrm{mmol} / \mathrm{mol}$ creatinine]). Results from follow-up analyses are presented in Table II. Adenosine levels were normal and $2 '$-deoxyadenosine was undetectable in the DBSs from the 2 healthy carriers (siblings of patients with early-onset ADA-SCID, Table I).

\section{TREC analysis}

Normal TREC levels were found (Table I) in DBSs collected at birth from patients $\mathrm{P} 1$ to P3. TRECs were undetectable in DBSs or whole blood from patients $\mathrm{P} 1$ to $\mathrm{P} 3$ at the time of diagnosis, as well as in 3 DBSs collected at birth from patients with early-onset ADA-SCID. Quantitative real-time PCR analysis demonstrated normal TREC levels in the first and second DBSs (collected at birth and on the fifth day of life) from patient P4 (Table II). TREC levels were normal in the DBSs collected at birth from the 2 healthy carriers (Table I).

\section{Genetic analysis}

Results from sequence analysis of the $A D A$ gene in patients $\mathrm{P} 1$ to $\mathrm{P} 3$, the patients with early-onset ADA-SCID, and patient $\mathrm{P} 4$ are shown in Table I. All variants found in patients P1 to P3 were previously described. ${ }^{8,9}$ Patient P4 had 2 missense substitutions in $A D A$ : a previously described heterozygous substitution of c. $43 \mathrm{C}>\mathrm{G}$ in exon 2, which results in His15Asp and was associated with ADA-SCID in the Human Gene Mutation Database, ${ }^{8}$ and the heterozygous substitution c.149T $>C$ in exon 3, which results in Ile50Thr. The latter variant was not detected in 200 Italian subjects without ADA-SCID, excluding the possibility that it is a polymorphism that is not associated with disease. Patient P4's mother and father were found to be heterozygous carriers of the variants in exons 2 and 3, respectively.

Two sequence homology-based tools (Sort Intolerant from Tolerant [SIFT] and Polymorphism Phenotype [PolyPhen]) were used to predict the effects of the nonsynonymous polymorphisms found in patient P4. SIFT predicted that Ile50Thr in exon 3 would not be tolerated, and PolyPhen reported that it would disrupt the ligand-binding site. However, these predictions could be imprecise, and therefore the effects of this substitution on the activity of
ADA were further analyzed (see "Additional characterization of patient P4" section).

\section{ADA activity}

The activities of ADA in cells from patients with early- or delayed-onset disease and negative control subjects are presented in Table I.

\section{Lymphocyte subset, immunoglobulin, and proliferation levels}

Subsets of lymphocytes in blood samples collected from patient $\mathrm{P} 4$ at the first and second follow-up visits were analyzed by using flow cytometry (to identify $\mathrm{CD}^{+}, \mathrm{CD} 4^{+}, \mathrm{CD} 8^{+}, \mathrm{CD} 19^{+}$, $\mathrm{CD}^{-}{ }^{-} \mathrm{CD} 16^{+} \mathrm{CD}^{+} 6^{+}, \mathrm{CD} 31^{+}, \mathrm{CD} 45 \mathrm{RA}, \mathrm{CD} 45 \mathrm{RO}, \mathrm{CD} 62$ ligand, and $\mathrm{CD} 11 \mathrm{a}^{\mathrm{dim}}$ cells). Proliferation was analyzed with PHA or CD3/ CD28 and carboxyfluorescein diacetate succinimidyl ester in flow cytometry. Values were found to be normal. No data were available for patients $\mathrm{P} 1$ to $\mathrm{P} 3$ at birth, but patient $\mathrm{P} 1$ had mild lymphopenia at 2 months of age that remained stable until 7 years of age. ${ }^{14}$ Patient P4 had normal serum immunoglobulin levels when she was 25 days old, and patient P1 had normal levels during the 5-year follow-up period from the second year of age to the time of diagnosis.

\section{Additional characterization of patient P4}

ADA activity. We expressed the ADA variant of patient P4 (Ile50Thr) in E coli by using a previously described method. ${ }^{8}$ We found that it had $29.5 \%$ of the activity of ADA from healthy subjects. On the basis of data from Arredondo-Vega et al, ${ }^{8}$ this level of enzymatic activity placed patient P4 at the upper limit of the range for activity group IV, which includes variants found in only children and adults with a partial ADA deficiency phenotype. However the half-life of the Ile50Thr variant at $40^{\circ} \mathrm{C}$ was about $17 \mathrm{~min}$ utes, whereas ADA from healthy subjects lost less than $2 \%$ of its activity after 2 hours at $40^{\circ} \mathrm{C}$, indicating that the variant is unstable.

ADA activity in separated red and white blood cells. The enzymatic activity of ADA in separate red or white blood cells was determined in 2 different laboratories by using methods $\mathrm{B}$ and C. No enzymatic activity was found in separated red cells from patient $\mathrm{P} 4$, whereas enzymatic activities in white cells were found to be $3.3 \%$ and $4.2 \%$ of those of ADA from healthy subjects, respectively, at the 2 laboratories. Cells from patient $\mathrm{P} 4$ were found to have normal purine-nucleoside phosphorylase activity, tested as an internal control, at each laboratory. 
A

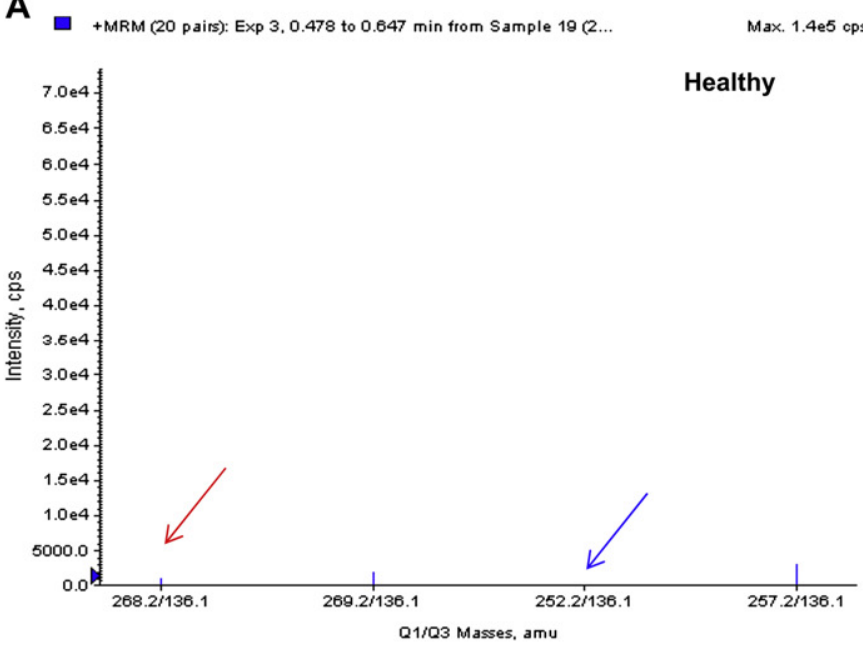

C

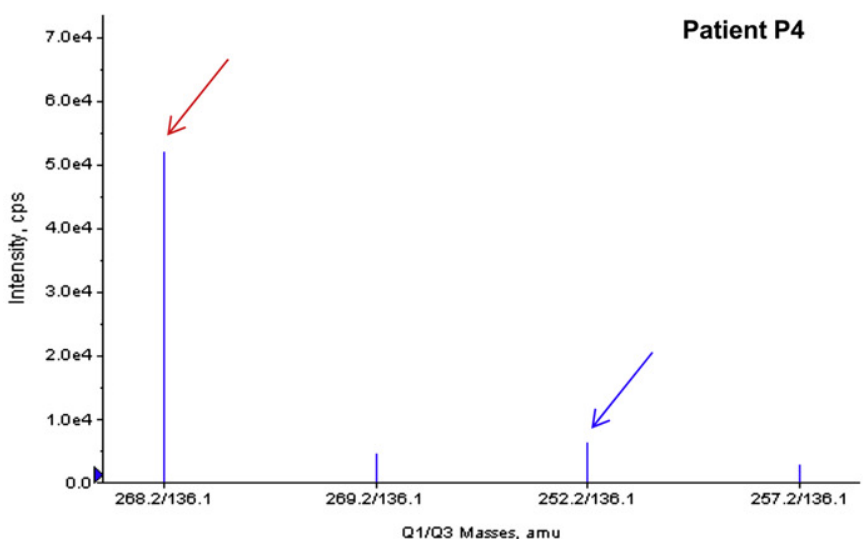

B

- +MRM (20 pairs): Exp 3, 0.478 to $0.647 \mathrm{~min}$ from Sample ...

$\operatorname{Max} .8 .1 \mathrm{e} 4 \mathrm{cps}$

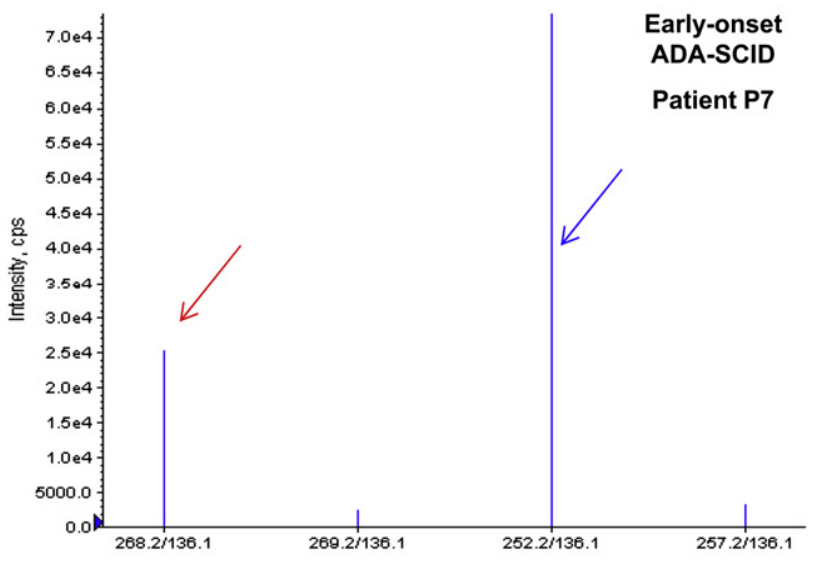

Q1/Q3 Masses, amv

D

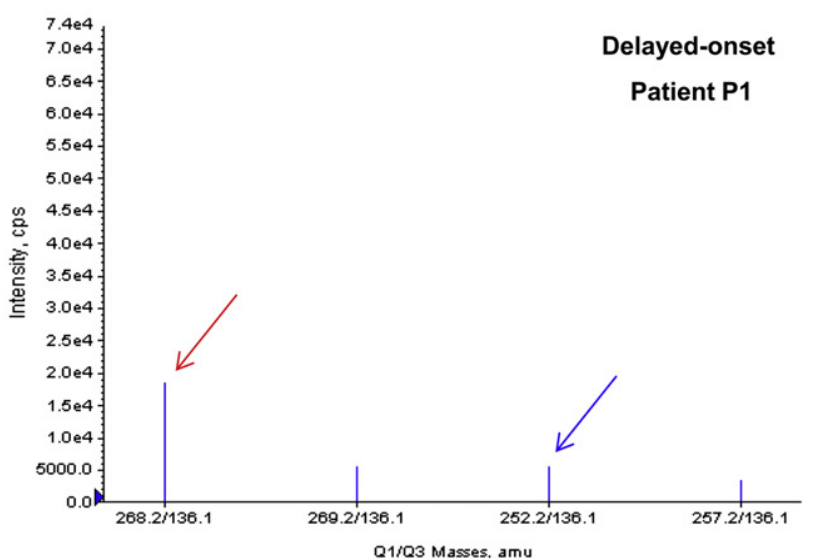

FIG 1. Tandem-MS and TREC analysis results. A-D, Metabolites adenosine and 2'-deoxyadenosine as they appear in a tandem-MS analysis of DBSs from a representative healthy newborn (Fig 1, $A$ ), a typical patient with early-onset ADA-SCID (Fig 1, B), a patient identified during the newborn screening procedure (P4; Fig 1, $C$ ), and a patient with delayed-onset ADA-SCID (P1; Fig 1, D). Adenosine is indicated by red arrows, and 2' -deoxyadenosine is indicated by blue arrows. In the same DBSs analyzed by using tandem-MS, TRECs were not detected in patients with early-onset ADA deficiency, whereas they were present at normal levels in a patient with delayed-onset ADA deficiency (P1), a patient found to be deficient in adenosine and 2 '-deoxyadenosine in a newborn screening program (P4), and a negative control subject. TREC levels are included in Table I.

dAXP levels in red blood cells. dAXP levels in red blood cells of patient $\mathrm{P} 4$ were tested simultaneously at 2 different laboratories in both frozen and thawed cells obtained when patients were 7 months old. dAXP levels measured at the 2 laboratories were 76 and $82 \mathrm{nmol} / \mathrm{mL}$, respectively, which is at the low end of the range observed in patients with a late-onset phenotype. ${ }^{8}$ However, the dAXP in the red cells from patient P4 accounted for only $3.3 \%$ of total adenine nucleotides, a value less than that usually detected in patients with late-onset disease and that has been reported only once in a patient with ADA-SCID at 6 years of age (M. S. Hershfield, personal observation in $>200$ patients with ADA deficiency).

\section{DISCUSSION}

Clinical findings, increased levels of adenosine and $2^{\prime}$-deoxyadenosine, and sequence analysis of $A D A$ led to a diagnosis of delayed-onset ADA deficiency in patients P1 to P3. However, their metabolic abnormalities (increased levels of adenosine and 2 '-deoxyadenosine) were already present at birth and could have been easily identified by using tandem-MS analysis of DBSs collected in a newborn screening program. Analysis of the same DBSs for TRECs did not identify any defect. We therefore propose that delayed-onset ADA-SCID can be identified in newborns through screening programs that include tandem-MS but not by TREC analysis. Further confirmation of these findings is now ongoing in our laboratories.

P4 was found to have increased adenosine and 2'-deoxyadenosine levels in a newborn screening program. These findings, along with sequence analysis of $A D A$, indicated that the patient had ADA deficiency, although TREC levels were normal (as for patients P1-P3). Both the SIFT and PolyPhen software predicted deleterious effects of the $A D A$ variant carried by patient P4, which encoded a I50T substitution. However, these software 


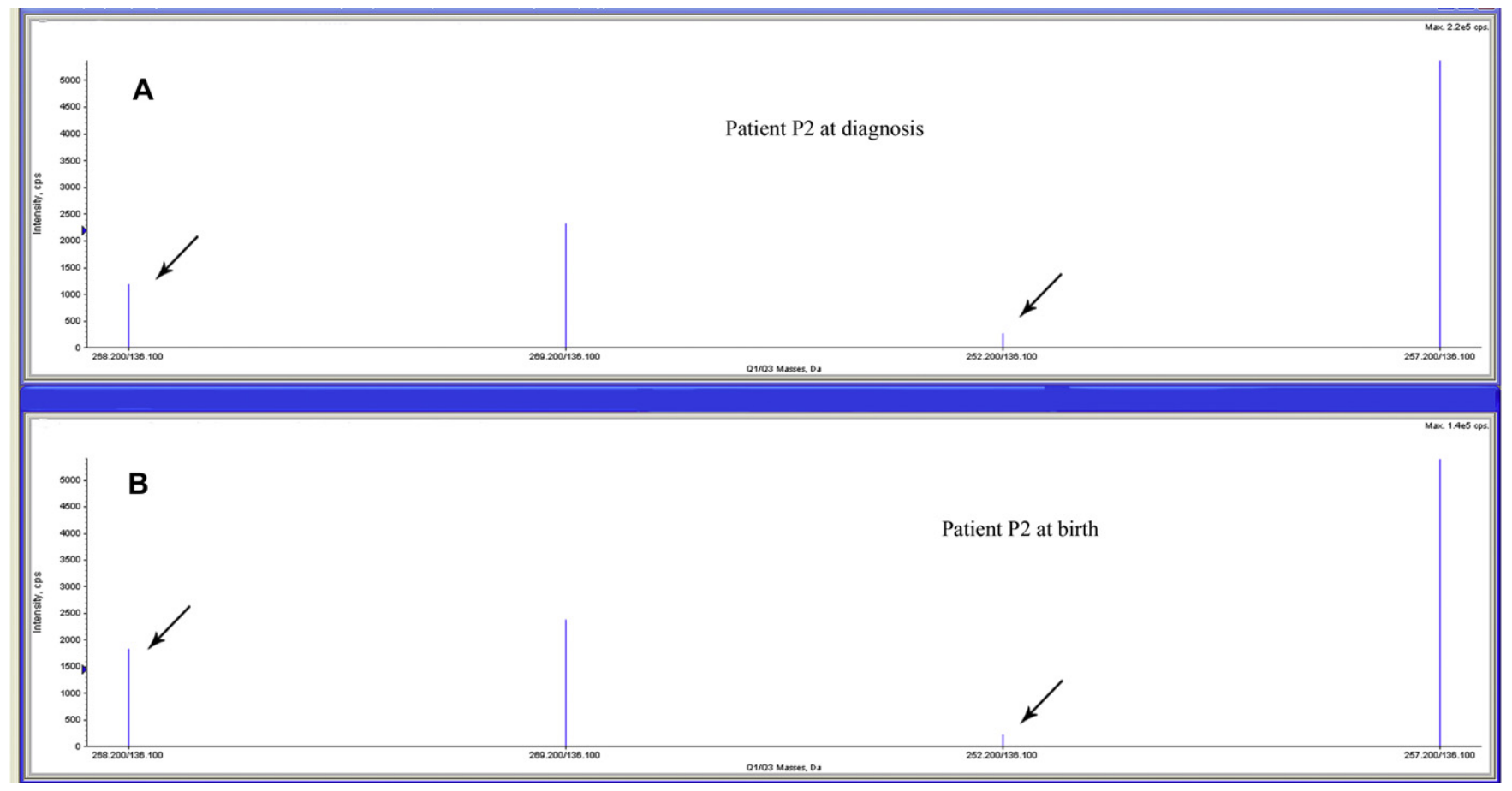

FIG 2. Tandem-MS analysis of patient $P 2$. A and B, Metabolites adenosine and 2 '-deoxyadenosine as they appear in a tandem-MS analysis of DBSs taken at diagnosis (Fig 2, A) or at birth (Fig 2, B) from a patient with delayed-onset disease (P2). Adenosine is indicated by red arrows, and 2'-deoxyadenosine is indicated by blue arrows.

systems can make inaccurate predictions, and the threshold levels of adenosine and $2{ }^{\prime}$-deoxyadenosine required to cause immune dysfunction are not known. We extensively analyzed patient P4 to determine her disease phenotype. Red blood cells from patient $\mathrm{P} 4$ were found to have no ADA enzyme activity, and white blood cells were found to have levels of activity that were about $3 \%$ to $4 \%$ of normal values. According to previous reports, ${ }^{8,18,20}$ healthy subjects with partial ADA deficiency have levels of ADA activity in white cells that are $5 \%$ to $70 \%$ of those of control subjects, whereas patients with SCID have levels of ADA activity that are less than $1 \%$ to $2 \%$ of those of control subjects.

The ADA variant carried by patient $\mathrm{P} 4$ was expressed in E coli, and functional analysis demonstrated its enzymatic activity to be $29.5 \%$ that of control ADA, placing it in activity group IV. ${ }^{8}$ Immunodeficiency has not been reported in patients carrying ADAs from activity group IV. ${ }^{8}$ No missense mutations that reduce enzyme activity have been previously observed in exon 3 of $A D A$, the location of patient P4's I50T substitution. This ADA variant was found to be unstable, with a half-life of only 17 minutes at $40^{\circ} \mathrm{C}$. However, most missense variants found in patients with ADA deficiency are unstable, including those found in patients with late-onset or partial deficiency. ${ }^{8,19,20,22,23}$ It is difficult to predict how ADA instability will affect patient P4, especially in situations of febrile infections.

To evaluate the in vivo function of ADA, we measured dAXP concentrations in red cells from patient P4. Levels of greater than $70 \mathrm{nmol} / \mathrm{mL}$, as confirmed in 2 different laboratories, would be at the low range found in patients with a late-onset phenotype. ${ }^{8}$ On the other hand, dAXP accounted for only $3.3 \%$ of total adenine nucleotides, indicating a partial form of ADA deficiency. ${ }^{8,22,24-26}$ Therefore on the basis of ADA activity, dAXP levels and percentages in patient $\mathrm{P} 4$ appear to border between partial and late-onset ADA deficiency.

Delayed and late-onset forms of ADA-SCID are well known, but these patients have not been identified in the preinfection period. At present, a diagnosis of delayed- or late-onset ADA deficiency can only be made when patients present with recurrent infections and sometimes autoimmunity. Blood cell numbers, immunoglobulin levels, and lymphocyte proliferation should be measured; recent thymic emigrants should be assessed; and adenosine metabolites should be quantified in the routine analysis of patients of any age with clinical signs of immunologic disorders. However, by the time a diagnosis is made, patients might already have permanent organ damage, as for patients P1 to P3. Our study found that patients with delayed-onset ADA-SCID have normal thymic function at birth and that thymic involution occurs over time.

We propose that tandem-MS can be used to identify newborns with not only early-onset ${ }^{12}$ but also delayed- and probably lateonset ADA deficiencies. Analysis of ADA metabolites by means of tandem-MS would have a low cost ${ }^{12}$ in most laboratories that already screen newborns by using tandem-MS. Actually, laboratories that perform tandem-MS with hydrazine could be able to also analyze adenosine and 2 -deoxyadenosine at no extra cost. The method could also be used, at no extra cost, in laboratories that substitute water for hydrazine. Further studies are needed to determine the correct time to begin therapy in infants found to have delayed- or late-onset ADA deficiency.

Clinical and biochemical follow-up analyses of patient $\mathrm{P} 4$ are underway. The low percentage of dAXP detected, as well as the normal adenosine and 2 '-deoxyadenosine values observed when patient P4 was 24 days old, might indicate she could remain immunocompetent for many years and possibly forever. However, characterization of her ADA activity and metabolic features 
indicate a disease phenotype that lies between late-onset and partial ADA deficiency; it is difficult to predict whether the disease will progress to a late-onset phenotype. Detection of abnormal adenosine metabolite levels in newborns identifies patients that should be followed and monitored for symptoms of ADA-SCID.

We do not have data on the activity of ADA; adenosine, $2^{\prime}$ deoxyadenosine, or dAXP levels; or immune function of patients P1 to $\mathrm{P} 3$ for the period between birth and diagnosis. Therefore we cannot predict whether normalization of adenosine and 2 '-deoxyadenosine levels found in patient P4's blood after the 25 th day of life will result in a symptom-free life or only represent a "honeymoon" period.

Patients P1 to P3 had severe immunodeficiency; their severe recurrent infections and lung and thymic damage could have been avoided through early identification with tandem-MS analysis of DBSs. Once a newborn is identified to have ADA metabolite abnormalities in a screening program, he or she should undergo extensive characterization of ADA activity, dAXP levels and percentages, and immune function to determine the disease phenotype. The patient should then be followed to determine when the first symptoms appear, so that treatment can begin before damage occurs. The actual aims of screening programs are not to make diagnoses but to identify patients who need further characterization with specific tests and clinical follow-up.

Most newborn screening programs for SCID are based on quantitative analysis of TRECs. However, we recommend the inclusion of tandem-MS analysis of adenosine and 2 -deoxyadenosine, which is inexpensive ${ }^{12}$ and can identify newborns with severe and hypomorphic variants of ADA, who might have SCID later in life. Moreover, tandem-MS can be useful in countries that cannot afford TREC analysis but are already using tandem-MS to screen for other metabolic diseases.

Clinical implications: Tandem-MS to measure adenosine and 2'-deoxyadenosine levels, but not quantification of TRECs, can identify newborns with delayed-onset ADA-SCID. Screening programs that include tandem-MS analysis could identify newborns with delayed-onset phenotype before symptoms appear, and therefore they can be treated before they have serious organ damage and infections.

\section{REFERENCES}

1. Notarangelo LD. Primary immunodeficiencies. J Allergy Clin Immunol 2010; 125(suppl 2):S182-94

2. Wilson JMG, Jungner G. Principles and practices of screening for disease. WHO Report no. 34, 1968. Available at: http://whqlibdoc.who.int/php/WHO_PHP_34. pdf. Accessed December 17, 2012.

3. Health Resources and Services Administration. Secretary's Advisory Committee on Heritable Disorders in Newborns and Children. Resolution from the 20th meeting, Washington. DC, January 22. 2010. Available at: http://www.hrsa.gov/ advisorycommittees/mchbadvisory/heritabledisorders/meetings/twentieth/minutes. pdf. Accessed December 17, 2012

4. Puck JM. Laboratory technology for population-based screening for severe combined immunodeficiency in neonates: the winner is T-cell receptor excision circles. J Allergy Clin Immunol 2012;129:607-16.

5. Comeau AM. Guidelines for implementation of population-based newborn screening for severe combined immunodeficiency. J Inherit Metab Dis 2010;33:273-81.
6. Sauer AV, Aiuti A. New insights into the pathogenesis of adenosine deaminasesevere combined immunodeficiency and progress in gene therapy. Curr Opin Allergy Clin Immunol 2009;9:496-502.

7. Sanchez JJ, Monaghan G, Børsting C, Norbury G, Morling N, Gaspar HB. Carrier frequency of a nonsense mutation in the adenosine deaminase (ADA) gene implies a high incidence of ADA-deficient severe combined immunodeficiency (SCID) in Somalia and a single, common haplotype indicates common ancestry. Ann Hum Genet 2007;71:336-47.

8. Arredondo-Vega FX, Santisteban I, Daniels S, Toutain S, Hershfield MS. Adenosine deaminase deficiency: genotype-phenotype correlations based on expressed activity of 29 mutant alleles. Am J Hum Genet 1998;63:1049-59.

9. Felgentreff K, Perez-Becker R, Speckmann C, Schwarz K, Kalwak K, Markelj G, et al. Clinical and immunological manifestations of patients with atypical severe combined immunodeficiency. Clin Immunol 2011;141:73-82.

10. Gaspar HB. Bone marrow transplantation and alternatives for adenosine deaminase deficiency. Immunol Allergy Clin North Am 2010;30:221-36.

11. Gaspar HB, Aiuti A, Porta F, Candotti F, Hershfield MS, Notarangelo LD. How I treat ADA deficiency. Blood 2009; 114:3524-32.

12. Azzari C, la Marca G, Resti M. Neonatal screening for severe combined immunodeficiency caused by an adenosine deaminase defect: a reliable and inexpensive method using tandem mass spectrometry. J Allergy Clin Immunol 2011;127:1394-9.

13. Chace DH. Mass spectrometry in newborn and metabolic screening: historical perspective and future directions. J Mass Spectrom 2009;44:163-70.

14. Speckmann C, Neumann C, Borte S, la Marca G, Sass JO, Wiech E, et al. Delayedonset adenosine deaminase deficiency: strategies for an early diagnosis. J Allergy Clin Immunol 2012;130:991-4.

15. la Marca G, Malvagia S, Pasquini E, Innocenti M, Fernandez MR, Donati MA, et al The inclusion of succinylacetone as marker for tyrosinemia type I in expanded newborn screening programs. Rapid Commun Mass Spectrom 2008;22:812-8.

16. la Marca G, Casetta B, Malvagia S, Pasquini E, Innocenti M, Donati MA, et al Implementing tandem mass-spectrometry as a routine tool for characterizing the complete purines and pyrimidines metabolic profile in urine samples. J Mass Spectrom 2006;41:1442-52

17. Fairbanks LD, Simmonds HA, Duley JA, Gaspar HB, Flood T, Steward CA. ADA activity and DATP levels in erythrocytes after bone marrow transplantation. Adv Exp Med Biol 2000;486:51-5

18. Arredondo-Vega FX, Kurtzberg J, Chaffee S, Santisteban I, Reisner E, Povey MS et al. Paradoxical expression of adenosine deaminase in $\mathrm{T}$ cells cultured from a patient with adenosine deaminase deficiency and combine immunodeficiency. J Clin Invest 1990;86:444-52

19. Hirschhorn R, Ellenbogen A. Genetic heterogeneity in adenosine deaminase (ADA) deficiency: five different mutations in five new patients with partial ADA deficiency. Am J Hum Genet 1986;38:13-25.

20. Arredondo-Vega FX, Santisteban I, Richard E, Bali P, Koleilat M, Loubser M, et al. Adenosine deaminase deficiency with mosaicism for a "second-site suppressor" of a splicing mutation: decline in revertant $\mathrm{T}$ lymphocytes during enzyme replacement therapy. Blood 2002;99:1005-13.

21. Baker MW, Grossman WJ, Laessig RH, Hoffman GL, Brokopp CD, Kurtycz DF, et al. Development of a routine newborn screening protocol for severe combined immunodeficiency. J Allergy Clin Immunol 2009;124:522-7.

22. Ariga T, Oda N, Sanstisteban I, Arredondo-Vega FX, Shioda M, Ueno H, et al. Molecular basis for paradoxical carriers of adenosine deaminase (ADA) deficiency that show extremely low levels of ADA activity in peripheral blood cells without immunodeficiency. J Immunol 2001;166:1698-702.

23. Hirschhorn R, Roegner V, Jenkins T, Seaman C, Piomelli S, Borkowsky W. Erythrocyte adenosine deaminase deficiency without immunodeficiency. Evidence for an unstable mutant enzyme. J Clin Invest 1979;64:1130-9.

24. Santisteban I, Arredondo-Vega FX, Kelly S, Loubser M, Meydan N, Roifman C, et al. Three new adenosine deaminase mutations that define a splicing enhancer and cause severe and partial phenotypes: implications for evolution of a $\mathrm{CpG}$ hotspot and expression of a transduced ADA cDNA. Hum Mol Genet 1995;4 2081-7.

25. Shovlin CL, Simmonds HA, Fairbanks LD, Deacock SJ, Hughes JM, Lechler RI, et al. Adult onset immunodeficiency caused by inherited adenosine deaminase deficiency. J Immunol 1994;153:2331-9.

26. Ozsahin H, Arredondo-Vega FX, Santisteban I, Fuhrer H, Tuchschmid P, Jochum W, et al. Adenosine deaminase deficiency in adults. Blood 1997;89:2849-55. 